\title{
Prevalencia y clasificación de enfermedad renal crónica en pacientes con diabetes mellitus tipo 2 en el centro comunitario de salud familiar Pantanosa, Frutillar
}

\author{
PABLO VILLARROEL R. ${ }^{1}$, XIMENA PARRA L. ${ }^{2}$, LEOPOLDO ARDILES A. ${ }^{3}$
}

\section{Frequency of chronic kidney disease among ambulatory patients with type 2 diabetes}

Background: Type 2 diabetes mellitus is the main cause of chronic kidney disease in developed countries. Aim: To study the prevalence of chronic kidney disease among adults with diabetes mellitus attended at a public primary health care clinic in southern Chile. Material and Methods: One hundred patients with type 2 diabetes mellitus, aged more than 15 years participated in this cross sectional study. Chronic kidney disease was defined as the presence of a urine albumin/creatinine ratio over 30 $\mathrm{mg} / \mathrm{g}$ or an estimated glomerular filtration rate of less than $60 \mathrm{~mL} / \mathrm{min} / 1,73 \mathrm{~m}^{2}$, detected in at least two opportunities, separated at least by three months. Results: Thirty four percent of participants had chronic kidney disease (17\% stage 1 or 2 and 17\% stage 3). Thirty percent of participants had an abnormal urinary albumin/creatinine ratio. Half of the patients with an estimated glomerular filtration rate below $60 \mathrm{~mL} /$ $\mathrm{min} / 1,73 \mathrm{~m}^{2}$, had a normal urinary albumin/creatinine ratio. Conclusions: The rates of chronic kidney disease in this group of diabetic patients are very similar to those reported elsewhere.

(Rev Med Chile 2012; 140: 287-294).

Key words: Diabetes mellitus; Kidney diseases; Renal insufficiency.

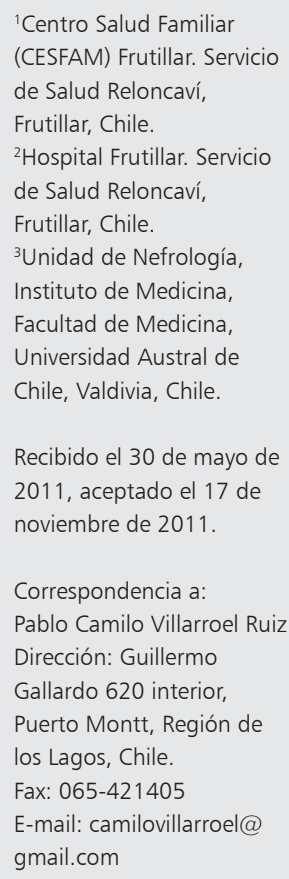

L a diabetes mellitus (DM) tipo 2 constituye la primera causa de casos nuevos de enfermedad renal crónica (ERC) en países desarrollados ${ }^{1}$ y la nefropatía diabética, la primera causa de ingreso a terapia dialítica crónica en nuestro país con $35 \%{ }^{2}$.

Los resultados de la Encuesta Nacional de Salud (ENS) 2010-2011 muestran un incremento en la prevalencia de DM tipo 2 en mayores de 15 años de $4,2 \%$ en 2003 a $9,4 \%{ }^{3}$. Estas cifras constituyen una preocupación debido al aumento esperable de patología cardiovascular y ERC asociada.

La evaluación y clasificación de la nefropatía diabética se realiza desde mucho tiempo según las etapas de Mogensen, basada en datos obtenidos de pacientes con diabetes mellitus tipo 1 y extrapolada a pacientes con DM tipo $2^{4}$. Esta clasificación consta de 5 etapas: 1) Hiperfiltración glomerular con hipertrofia renal; 2) Normoalbuminuria con engrosamiento de la membrana basal glomerular y expansión mesangial; 3) Microalbuminuria e hipertensión arterial; 4) Macroalbuminuria; 5) Insuficiencia renal crónica terminal (IRCt).

La microalbuminuria constituye el marcador precoz de desarrollo de nefropatía diabética y se ha establecido su progresión a macroalbuminuria aproximadamente en 10 años. A su vez, la macroalbuminuria, durante un período de 5 a 10 años determina el desarrollo de IRCt con la necesidad de terapia de sustitución renal ${ }^{5}$. Esto constituyó la principal razón de detección y tratamiento precoz de la nefropatía diabética durante mucho tiempo.

Actualmente, la declinación de la VFG cobra cada vez mayor importancia; no sólo por su 
progresión a IRCt, sino por su reciente reconocimiento como factor de riesgo cardiovascular independiente a valores inferiores de $60 \mathrm{~mL} /$ $\mathrm{min} / 1,73 \mathrm{~m}^{2}$, tanto en población general como en pacientes diabéticos ${ }^{6-8}$.

A su vez, estudios recientes han definido al cuociente albuminuria/creatininuria (A/C) como factor de riesgo cardiovascular adicional e independiente, y que aumenta progresivamente a partir de valores considerados tradicionalmente dentro del rango de la normalidad tales como $10 \mathrm{mg}$ de albúmina/gramo de creatininuria ${ }^{8}$.

Por otro lado, estudios utilizando la clasificación de Mogensen, muestran que la hiperfiltración glomerular y su asociación a largo plazo con el desarrollo de microalbuminuria e insuficiencia renal, no está tan clara ${ }^{9}$. Además, las fórmulas para calcular la velocidad de filtración glomerular estimada (VFGe) basadas en los niveles de creatinina sérica, tienen pobre correlación a valores $>$ $60 \mathrm{~mL} / \mathrm{min} / 1,73 \mathrm{~m}^{2}$ y los métodos más exactos, son engorrosos y costosos de realizar. Finalmente, la etapa de macroalbuminuria se ha asociado implícitamente con el inicio de la declinación de la VFG, sin embargo, se ha demostrado que esta declinación puede tener un comportamiento independiente de los niveles de albuminuria en pacientes diabéticos ${ }^{10,11}$.

Es así como la nueva clasificación de ERC publicada por la National Kidney Fundation (NKF) en el año $2002^{12}$ nace como alternativa, no sólo como un intento de unificar terminologías que permitan estandarizar los estudios clínicos y epidemiológicos, sino además por el reconocimiento de los dos elementos más importantes de este sistema de clasificación (albúmina en orina y VFGe) como nuevos factores de riesgo de mortalidad general $y$ cardiovascular.

En atención primaria, la DM tipo 2 constituye la patología crónica no transmisible más importante bajo control y es en este lugar donde se deben tomar todas la iniciativas de prevención, pesquisa, tratamiento y derivación de la ERC. A pesar de lo anterior, esta se encuentra aún ampliamente subdiagnosticada ${ }^{13}$ con todas las consecuencias pronósticas y económicas que esto implica.

Dado el reconocido impacto de la ERC como factor de riesgo cardiovascular, su alta prevalencia e incidencia en los pacientes con DM tipo 2 y la escasez de publicaciones a nivel local, particularmente en atención primaria, es que se hace impres- cindible tener una aproximación de la envergadura de este problema de salud pública.

El objetivo de este trabajo fue el conocer la prevalencia y clasificación de la ERC de acuerdo a los criterios de clasificación de la K/DOQI en este subgrupo de riesgo, caracterizar a los pacientes que presentan VFGe inferior de $60 \mathrm{~mL} / \mathrm{min} / 1,73$ $\mathrm{m}^{2}$ y demostrar la independencia que tienen en ciertas oportunidades la VFGe y los niveles de albuminuria, justificando el uso de este sistema de clasificación de ERC en pacientes con DM tipo 2.

\section{Material y Método}

Se realizó un estudio epidemiológico descriptivo transversal, en el Centro Comunitario de Salud Familiar (CECOSF) Pantanosa de Frutillar, con una población a cargo de 4.876 inscritos, de alto riesgo social, bajo nivel educacional y económico. El centro asistencial es de implementación básica y bajo nivel de complejidad. Se incluyó en el estudio la totalidad de los pacientes mayores de 15 años con DM tipo 2 en control ( $n=100)$. Los datos fueron obtenidos de la ficha clínica correspondiente al programa cardiovascular del centro asistencial.

La variables estudiadas fueron características sociodemográficas (edad, género, nivel educacional) antecedentes personales (tabaquismo, sedentarismo) parámetros antropométricos (talla peso, índice de masa corporal), comorbilidades asociadas (hipertensión arterial, hipercolesterolemia, hipertrigliceridemia, eventos cardiovasculares previos) y resultados de exámenes de laboratorio (colesterol total, colesterol HDL, triglicéridos, creatinina sérica, cuociente albuminuria/ creatininuria, proteinuria semicuantitativa, glucosuria semicuantitativa, Hemoglobina glicosilada).

El nivel educacional se clasificó en analfabetismo (sin antecedentes de instrucción formal), nivel educacional bajo (menos de 8 años de instrucción formal), medio (entre 8 y 12 años de instrucción formal) y alto (más de 12 años de instrucción formal).

Tabaquismo se definió como el consumo dentro del último mes de cualquier número de cigarrillos. Sedentarismo se definió como la práctica de ejercicio físico menor a 30 minutos 3 veces por semana o su ausencia.

El peso y la talla fue medido en kilos y metros respectivamente mediante báscula calibrada de 
pie con tallímetro. El índice de masa corporal (IMC) se calculó mediante la división del peso en kilogramos por la talla en metros al cuadrado clasificando en: normal 20-24,9 sobrepeso 25-29,9, obesidad 30-39,9 y obesidad mórbida 40 o más.

Se definió la presencia de hipertensión arterial (HTA) mediante el registro previo de valores superiores a 140/90 $\mathrm{mmHg}$ dos o más veces separadas por varias semanas, medida mediante esfigmomanómetro manual por personal capacitado o autorreferencia de la enfermedad asociado a uso actual de antihipertensivos.

Hipercolesterolemia se definió como la presencia de colesterol total mayor a $200 \mathrm{mg} / \mathrm{dl}$ en al menos una ocasión o el antecedente de tratamiento con estatinas.

Hipertrigliceridemia se definió como la presencia de triglicéridos mayor a $150 \mathrm{mg} / \mathrm{dl}$ en al menos una ocasión o el antecedente de tratamiento con fibratos.

Evento cardiovascular previo se definió como el antecedente de infarto agudo al miocardio, cardiopatía coronaria, accidente vascular encefálico, insuficiencia cardiaca o enfermedad vascular periférica, referido por el paciente o que constara en ficha clínica.

Los análisis de laboratorio fueron realizados en el Laboratorio del Hospital de Frutillar. El colesterol total fue medido mediante prueba enzimática fotométrica CHOD-PAP. El nivel de triglicéridos fue medido mediante prueba enzimática calorimétrica utilizando glicerol-3-fosfato-oxidasa. El colesterol HDL fue medido mediante método Fosfotungstínico-Magnesio. La cuantificación de proteinuria y glucosuria semicuantitativa se realizó mediante cintas reactivas. La hemoglobina glicosilada (HbA1c) se midió mediante espectrofotometría de reflectancia.

La medición de creatinina sérica y urinaria fue realizada mediante método de Jaffé cinético y no fue calibrada mediante el método de referencia de espectrometría de masa por dilución isotópica (IDMS). La cuantificación de albúmina en orina fue realizado por técnica inmunológica mediante el kit comercial "APTEC DIAGNOSTIC".

La VFGe se calculó mediante la fórmula MDRD-4 (cuatro variables): VFGe ( $\mathrm{ml} / \mathrm{min} /$ $\left.1,73 \mathrm{~m}^{2}\right)=186 \mathrm{x}$ creatinina sérica ${ }^{-1,154}(\mathrm{mg} / \mathrm{dl}) \mathrm{x}$ edad $^{-0,203} \times 0,742$ (si mujer) x 1,21 (si afroamericano).

Para clasificar la ERC en etapas, se consideró como 1 y 2 a los sujetos con cuociente $\mathrm{A} / \mathrm{C}$ igual o mayor de $30 \mathrm{mg} / \mathrm{dl}$ y VFGe $\geq 60 \mathrm{~mL} / \mathrm{min} / 1,73$ $\mathrm{m}^{2}$; las etapas 3a, 3b, 4 y 5 incluyeron pacientes con VFGe entre 45-59, 30-44, 15-29 y menor de 15 (o diálisis crónica) $\mathrm{mL} / \mathrm{min} / 1,73 \mathrm{~m}^{2}$, respectivamente, sin importar el valor de la excreción de albúmina urinaria.

Se definió entonces ERC como la presencia de cuociente A/C igual o superior a $30 \mathrm{mg} / \mathrm{g}$ en dos tomas de primera orina de la mañana, repetidas y separadas al menos por 3 meses (en ausencia de infección urinaria o cuadro intercurrente febril) y/o la presencia de VFGe inferior a $60 \mathrm{~mL} / \mathrm{min} / 1,73$ $\mathrm{m}^{2}$, persistente en dos oportunidades separadas al menos por 3 meses.

\section{Estadística}

Se realizó mediante el programa SPSS 17.0. Los resultados de las variables continuas se expresaron en promedio y desviación estándar de la media. Los resultados de las variables categóricas se expresaron en porcentajes. Las diferencias entre variables continuas fueron analizadas mediante la prueba $\mathrm{T}$ de Student y la diferencia entre proporciones mediante test de $\chi^{2}$.

\section{Resultados}

Las características sociodemográficas y clínicas de los pacientes en estudio se encuentran en la Tabla 1. Se observa una mayor proporción de pacientes de sexo femenino en control (mujeres 76\% vs hombres 24\%). El nivel educacional muestra que $85 \%$ del grupo en estudio presentó menos de 8 años de estudio formal. El sedentarismo y el tabaquismo fueron de $96 \%$ y $20 \%$ respectivamente. El promedio de IMC fue de 32,5 $\pm 5,3$ con $72 \%$ de pacientes con IMC igual o superior a 30 . Sólo $8 \%$ de los pacientes presentó IMC dentro de rangos normales. El 73\% presentó HTA asociada, 74\% hipercolesterolemia y $49 \%$ hipertrigliceridemia. El 10\% tuvo antecedentes de algún evento cardiovascular previo. El promedio general de HbA1c fue de $7,4 \pm 1,3 \%$ y $36 \%$ presentó glucosuria semicuantitativa en alguno de sus grados.

El promedio general de cuociente $\mathrm{A} / \mathrm{C}$ fue de $37,8 \pm 86,8 \mathrm{mg} / \mathrm{g}$ y la prevalencia de cuociente $\mathrm{A} / \mathrm{C}$ igual o mayor de $30 \mathrm{mg} / \mathrm{g}$ fue de $23 \%$. El $4 \%$ del total se presentó con valores de cuociente $\mathrm{A} / \mathrm{C}$ en rango de macroalbuminuria (> $300 \mathrm{mg} / \mathrm{g}$ ). Res- 
Tabla 1. Características Sociodemográficas, Clínicas y de Laboratorio de pacientes con DM tipo 2 bajo en el CECOSF Pantanosa, Frutillar $(n=100)$

\begin{tabular}{|c|c|c|}
\hline Edad & Promedio, años (DS) & $57,3 \pm 12,4$ \\
\hline \multirow[t]{5}{*}{ Grupos etarios } & 15 - 29 años & $1 \quad(1 \%)$ \\
\hline & 30 - 44 años & $14(14 \%)$ \\
\hline & $45-59$ años & $45(45 \%)$ \\
\hline & 60 - 74 años & $33(33 \%)$ \\
\hline & 75 y más años & $7 \quad(7 \%)$ \\
\hline \multirow[t]{2}{*}{ Sexo } & Femenino & $76(76 \%)$ \\
\hline & Masculino & $24(24 \%)$ \\
\hline \multirow[t]{4}{*}{ Nivel educacional } & Analfabetos & $17(17 \%)$ \\
\hline & N. educacional bajo & $68(68 \%)$ \\
\hline & N. educacional medio & $15(15 \%)$ \\
\hline & N. educacional alto & 0 \\
\hline \multirow[t]{2}{*}{ Ant. personales } & Tabaquismo & $20(20 \%)$ \\
\hline & Sedentarismo & $96(96 \%)$ \\
\hline \multirow{6}{*}{$\begin{array}{l}\text { Parámetros } \\
\text { antropométricos }\end{array}$} & Peso, promedio Kg (DS) & $76,2 \pm 15,6$ \\
\hline & Índice de Masa Corporal (IMC) & $32,5 \pm 5,3$ \\
\hline & Normal (IMC 20 - 24,9) & $8 \quad(8 \%)$ \\
\hline & Sobrepeso (IMC 25 - 29,9) & $20(20 \%)$ \\
\hline & Obesidad (IMC 30 - 39,9) & $63(63 \%)$ \\
\hline & Obesidad mórbida (IMC $\geq 40$ ) & 9 (9\%) \\
\hline \multirow[t]{4}{*}{ Comorbilidades } & Hipertensión Arterial & $73(73 \%)$ \\
\hline & Hipercolesterolemia & $74(74 \%)$ \\
\hline & Hipertrigliceridemia & $49(49 \%)$ \\
\hline & Morbilidad Cardiovascular ${ }^{1}$ & $10(10 \%)$ \\
\hline \multirow[t]{4}{*}{ Perfil lipídico } & Colesterol Total (mg/dl) & $195,4 \pm 55,8$ \\
\hline & Colesterol HDL (mg/dl) & $55,9 \pm 13$ \\
\hline & Colesterol LDL(mg/dl) & $108,8 \pm 41,8$ \\
\hline & Triglicéridos (mg/dl) & $180,5 \pm 107,2$ \\
\hline Control metabólico & $\begin{array}{l}\text { Hemoglobina Glicosilada } \\
\text { (HbA1c) mg/dl }\end{array}$ & $7,4 \pm 1,3$ \\
\hline Tratamiento & Uso de insulina & $21(21 \%)$ \\
\hline \multirow[t]{2}{*}{ Exs. de orina } & $\begin{array}{l}\text { Proteinuria positiva } \\
\text { (semicuantitativa) }\end{array}$ & 17 (17\%) \\
\hline & $\begin{array}{l}\text { Glucosuria positiva } \\
\text { (semicuantitativa) }\end{array}$ & 36 (36\%) \\
\hline
\end{tabular}

1. accidente vascular encefálico, Infarto agudo al miocardio, Insuficiencia cardiaca congestiva. pecto a la VFGe, $17 \%$ presentó valores inferiores a $60 \mathrm{ml} / \mathrm{min} / 1,73 \mathrm{~m}^{2}(9 \%$ entre 45 y 60 y $8 \%$ entre 30 y 44$)$. No se detectó pacientes con valores de VFGe $<30 \mathrm{ml} / \mathrm{min} / 1,73 \mathrm{~m}^{2}$.

De acuerdo a los criterios diagnósticos de la National Kidney Fundation, $34 \%$ presentó ERC en alguna de sus etapas. En relación al total de pacientes del estudio $17 \%$ se encontró en etapa 1 ó 2 y $17 \%$ en etapa 3 ( $9 \%$ en etapa $3 a$ y $8 \%$ en etapa $3 b)$. No hubo pacientes en etapa 4 ó 5 (Tabla 2).

Al analizar las características sociodemográficas, clínicas y de laboratorio que se observaron en pacientes con $\mathrm{VFGe}<60 \mathrm{ml} / \mathrm{min} / 1,73 \mathrm{~m}^{2}$, se encontró que el grupo con valores inferiores a $60 \mathrm{ml} / \mathrm{min} / 1,73 \mathrm{~m}^{2}$ presentó un promedio de edad de $67+$ 9,4 años y el grupo con valores igual o superiores a $60 \mathrm{ml} / \mathrm{min} / 1,73 \mathrm{~m}^{2}$, un promedio de $55+11,9$ años $(\mathrm{p}<0,05)$. Adicionalmente, dentro del grupo con VFGe inferior de $60 \mathrm{ml} / \mathrm{min} / 1,73 \mathrm{~m}^{2}$, $35,3 \%$ eran analfabetos comparado con un $13,3 \%$ del grupo con valores $\geq$ $60 \mathrm{ml} / \mathrm{min} / 1,73 \mathrm{~m}^{2}$, diferencia que no tuvo significación estadística (Tabla 3 ).

A su vez dentro del grupo con valores inferiores a $60 \mathrm{ml} / \mathrm{min} / 1,73$ $\mathrm{m}^{2}$ el $100 \%$ presentó HTA asociada comparado con el $67,5 \%$ de aquellos con VFGe superior a $60 \mathrm{ml} / \mathrm{min} / 1,73$ $\mathrm{m}^{2}(\mathrm{p}<0,05)$. Sólo $2(11,8 \%)$ de los pacientes con VFGe inferior a $60 \mathrm{ml} /$ $\mathrm{min} / 1,73 \mathrm{~m}^{2}$ presentaron glucosuria en alguno de sus grados, comparado con $34(41 \%)$ de aquellos con VFGe igual o superior a $60 \mathrm{ml} / \mathrm{min} / 1,73 \mathrm{~m}^{2}$ $(\mathrm{p}<0,05)$.

La Figura 1 muestra que entre los pacientes en etapa 1 y 2 , el $82,4 \%$ presentó microalbuminuria y $17,6 \%$ macroalbuminuria. En la etapa 3a, $88,9 \%$ de los pacientes presentó rangos de normoalbuminuria y $11,1 \%$ macroalbuminuria. No hubo pacientes en esta etapa con rangos de microalbuminuria. De los pacientes en etapa $3 b$, $37,5 \%$ presentó normoalbuminuria y 
Tabla 2. Clasificación de ERC, Albuminuria y Velocidad de filtración glomerular estimada (VFGe) en pacientes con DM Tipo 2 bajo control en el CECOSF Pantanosa, Frutillar $(n=100)$

\begin{tabular}{|c|c|c|}
\hline \multirow{4}{*}{$\begin{array}{l}\text { Cuociente } \\
\text { Albuminuria/Creatininuria (A/C) }\end{array}$} & A/C Promedio $(\mathrm{mg} / \mathrm{g})$ & $37,8 \pm 86,8$ \\
\hline & Número (\%) pacientes con $<30 \mathrm{mg} / \mathrm{g}$ & $77(77 \%)$ \\
\hline & Número (\%) pacientes con microalbuminuria (30 - 300 mg/g) & 19 (19\%) \\
\hline & Número (\%) pacientes con macroalbuminuria (> 300 mg/g) & $4(4 \%)$ \\
\hline \multirow[t]{3}{*}{ Distribución por VFGe } & Número pacientes (\%) con $\geq 60 \mathrm{ml} / \mathrm{min} / 1,73 \mathrm{~m}^{2}$ & $83(83 \%)$ \\
\hline & Número pacientes (\%) con $45-59 \mathrm{ml} / \mathrm{min} / 1,73 \mathrm{~m}^{2}(\%)$ & $9(9 \%)$ \\
\hline & Número pacientes (\%) con $30-44 \mathrm{ml} / \mathrm{min} / 1,73 \mathrm{~m}^{2}(\%)$ & $8(8 \%)$ \\
\hline \multirow[t]{3}{*}{ Clasificación ERC } & Número pacientes (\%) en Etapa 1 y 2 & $17(17 \%)$ \\
\hline & Número pacientes (\%) en Etapa 3a & $9(9 \%)$ \\
\hline & Número pacientes (\%) en Etapa 3b & $8(8 \%)$ \\
\hline
\end{tabular}

Tabla 3. Comparación de características sociodemográficas, clínicas y de laboratorio entre grupos de pacientes DM tipo 2 con VFGe $\geq 60 \mathrm{v} / \mathrm{s}<60 \mathrm{ml} / \mathrm{min} / 1,73 \mathrm{~m}^{2}$

\begin{tabular}{|lccc|}
\hline & VFGe $\geq \mathbf{6 0}$ & VFGe $<\mathbf{6 0}$ & p \\
$\mathrm{n}$ & 83 & 17 & \\
\hline Edad (años) & $55 \pm 11,9$ & $67 \pm 9,4$ & 0,0001 \\
\hline Femenino (\%) & $61(73,5)$ & $15(88,2)$ & NS \\
\hline Analfabeto (\%) & $11(13,3)$ & $6(35,3)$ & NS \\
\hline Tabaquismo (\%) & $19(22,9)$ & $1(5)$ & NS \\
\hline Sedentarismo (\%) & $80(96,4)$ & $16(88,8)$ & NS \\
\hline IMC (Kg/Mt²) & $32,6 \pm 5,4$ & $32,2 \pm 4,6$ & NS \\
\hline Hipertension Arterial (\%) & $56(67,5)$ & $17(100)$ & 0,01 \\
\hline Hipercolesterolemia (\%) & $61(73,5)$ & $13(76,5)$ & NS \\
\hline Hipertrigliceridemia (\%) & $38(45,8)$ & $11(64,7)$ & NS \\
\hline Morbilidad cardiovascular (\%) & $8(9,6)$ & $2(11,8)$ & NS \\
\hline Colesterol total (mg/dl) & $198 \pm 57,3$ & $183,3 \pm 47,4$ & NS \\
\hline Colesterol HDL (mg/dl) & $52,2 \pm 12,8$ & $50,3 \pm 13,7$ & NS \\
\hline Trigliceridos (mg/dl) & $181,5 \pm 113,1$ & $176,1 \pm 77$ & NS \\
\hline Creatinina sérica (mg/dl) & $0,88 \pm 0,14$ & $1,31 \pm 0,28$ & 0,0001 \\
\hline Hemoglobina glicosilada (\%) & $7,3 \pm 1,3$ & $7,3 \pm 1,1$ & NS \\
\hline Uso de insulina (\%) & $15(18,1)$ & $6(35,3)$ & NS \\
\hline Cuociente A/C (mg/g) & $34,8 \pm 84,9$ & $51,3 \pm 96,2$ & NS \\
\hline Glucosuria semicuantitativa (\%) & $34(41)$ & $2(11,8)$ & 0,04 \\
\hline Proteinuria semicuantitativa (\%) & $12(14,5)$ & $5(29,4)$ & NS \\
\hline
\end{tabular}

$62,5 \%$ microalbuminuria; cabe destacar que no se encontraron pacientes en esta etapa con rangos de macroalbuminuria y que de los 17 pacientes con VFGe < $60 \mathrm{ml} / \mathrm{min} / 1,73 \mathrm{~m}^{2}$, la mitad no presentó microalbuminuria.

\section{Discusión}

Nuestros resultados vienen a aportar datos útiles para conocer la prevalencia y características epidemiológicas de la ERC en pacientes diabéticos tipo 2 manejados en un consultorio de atención primaria en Chile.

Algunos elementos metodológicos deben ser considerados. Nuestro estudio está limitado por su diseño transversal, lo que no hace posible obtener información sobre la evolución de la ERC y determinar los factores relacionados con su progresión; en segundo lugar, el tamaño muestral no permite hacer análisis estadísticos de asociación más complejos y a su vez la características de la muestra, dada la alta proporción de mujeres y 


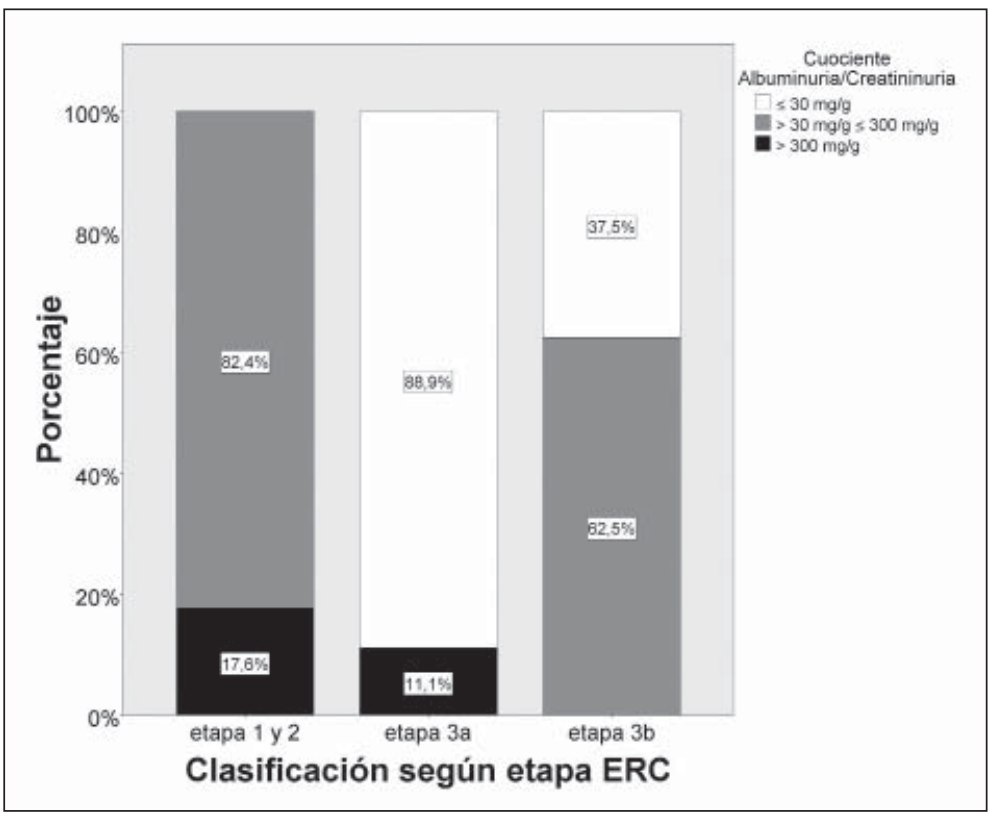

Figura 1. Distribución según nivel de Cuociente Albuminuria/Creatininuria (A/C) y Etapa de Clasificación de Enfermedad Renal Crónica. el bajo nivel educacional encontrado, restringen la extrapolación a otras realidades de atención primaria. Por último, la clasificación en etapa 1 y 2 fue realizada sólo mediante la pesquisa de cuociente $\mathrm{A} / \mathrm{C}$ y proteinuria semicuantitativa, no incluyéndose estudio de imágenes, histología u otros marcadores de injuria renal, lo que podría causar una subestimación de la verdadera prevalencia de ERC en estas etapas más precoces.

Con estas restricciones, nuestros datos permiten mostrar que la prevalencia de ERC en nuestros diabéticos adultos, de acuerdo al sistema de clasificación propuesto por la K/DOQI de la NKF, fue de $34 \%$, comparable con resultados encontrados en estudios realizados por el Kidney Early Evaluation Program (KEEP) aplicado en países como México donde mostró prevalencias de $35-38 \%{ }^{14}$ o aquéllos realizados en Japón con una prevalencia de $35 \%{ }^{15}$. La prevalencia de microalbuminuria del estudio NANHES III fue de $28,8 \%$ para pacientes con DM tipo $2^{16}$, muy cercano al $23 \%$ encontrado en nuestro estudio. En relación a la prevalencia de VFGe inferior a $60 \mathrm{ml} / \mathrm{min} / 1,73 \mathrm{~m}^{2}$, un estudio realizado en atención primaria en el extranjero mostró una prevalencia de $26 \%^{17}$. Nuestra prevalencia fue menor $(17 \%)$ pero hay que considerar que en ese estudio no fueron consideradas 2 mediciones de VFGe $<60 \mathrm{ml} / \mathrm{min} / 1,73 \mathrm{~m}^{2}$ persistentes en un intervalo de al menos 3 meses. Esto es reforzado por otro estudio que, utilizando nuestra misma estrategia de 2 mediciones, mostró $17,5 \%$ de VFGe inferior a $60 \mathrm{ml} / \mathrm{min} / 1,73 \mathrm{~m}^{2}$ en pacientes con DM tipo $2^{18}$, equivalente a nuestros hallazgos.

Se debe mencionar que la subdivisión en etapa 1 y 2 (que valora la presencia de marcadores de injuria renal en paciente con VFGe igual o superior a $60 \mathrm{ml} / \mathrm{min} / 1,73 \mathrm{~m}^{2}$ ) en atención primaria carece de aplicabilidad, debido a que la fórmulas de VFGe tienen una mala correlación con valores superiores a 60 , por lo cual se ha sugerido considerarla una sola etapa, salvo que se cuente con marcadores de función renal que presenten mejor correlación con valores superiores de VFG, como la Cistatina $\mathrm{C}^{19}$.

Dentro de los elementos relacionados con una reducción en la VFGe, se encuentran reconocidos la edad, la HTA asociada y la mala compensación metabólica. De acuerdo a lo anterior los grupos de riesgo para ERC a los cuales se recomienda realizar tamizaje de pesquisa según la K/DOQUI de la NKF incluyen los pacientes con Diabetes mellitus, Hipertensión arterial, edad mayor de 60 años y antecedentes familiares de $\mathrm{ERC}^{12}$.

La importancia de esta nueva clasificación es que sus dos dimensiones, la albuminuria y la VFGe, pueden presentar a su vez comportamientos de progresión estrechamente relacionados o bien se- 
guir cursos independientes entre sí. Los pacientes pueden tener disminución de la VFGe, asociado en forma previa con un incremento exponencial de la albuminuria, o bien tener una disminución de la VFGe sin tener aumento considerable de la albuminuria o incluso nunca salir de su condición de normoalbuminuria. Evidencias de este curso independiente aparecen ya en 1994 donde estudiando la evolución de la enfermedad renal en una cohorte de diabéticos tipo 1 y 2 se encontró que de 25 pacientes con disminución de la VFGe (con un seguimiento de 8-14 años) 13 no presentaron incremento significativo en los rangos de albúmina urinaria ${ }^{20}$.

Estudios más recientes en DM tipo 2, muestran que, del total de pacientes con VFG inferior a 60 $\mathrm{mL} / \mathrm{min} / 1,73 \mathrm{~m}^{2}, 30-40 \%$ no presenta excreción anormal de albúmina ${ }^{11,21}$, abriendo la discusión sobre la existencia de vías no albuminúricas de progresión de la ERC en pacientes diabéticos. En nuestro estudio queda en evidencia esta disrelación, ya que no todos los pacientes con VFGe más bajos presentaron valores de albuminuria altos, incluso, en más de la mitad de los pacientes con VFGe inferior de $60 \mathrm{~mL} / \mathrm{min} / 1,73 \mathrm{~m}^{2}$ no se detectó microalbuminuria. Esto cuestiona si la ausencia de albuminuria es suficiente argumento para descartar la presencia de ERC en pacientes con DM tipo 2; y abre en este grupo, la puerta a otras posibles causas de nefropatía, de gran prevalencia pero insuficientemente estudiadas como la secundaria a hipertensión arterial ${ }^{22}$.

Finalmente, esperamos que nuestros datos contribuyan al esfuerzo que se está realizando en nuestro país para conocer la real dimensión de la epidemia de enfermedad renal crónica que enfrentamos y así poder intervenir efectivamente reduciendo el peso económico y social con que ella nos amenaza.

\section{Referencias}

1. US Renal Data System. USRDS 2010 Annual Data Report. Atlas of end-stage renal disease in the United States. Bethesda (MD): National Institutes of Health, National Institute of Diabetes and Digestive and Kidney Diseases; 2004. Disponible en: http://www.usrds. org/2010/slides/indiv/1v2index.html

2. Poblete H. XXX Cuenta de hemodiálisis Crónica en Chile. Sociedad Chilena de Nefrología, Regis- tro de diálisis. Disponible en: http://www.nefro.cl/ index.php?option=com_phocadownload \&view=cat egory\&id=26:xxvii-congreso-chileno-de-nefrologia2010\&Itemid $=5$

3. Ministerio de Salud de Chile, Pontificia Universidad Católica de Chile. Resultados II Encuesta de Salud, Chile 2009-2010. Disponible en: http://www.redsalud.gov.cl/ portal/ url/item/99c12b89738d80d5e04001011e0113f8. pdf

4. Mogensen CE. Microalbuminuria, blood pressure and diabetic renal disease: origin and development of ideas. Diabetología 1999; 42 (3): 263-85.

5. Lambers Heerspink HJ, Gansevoort RT, Brenner BM, Cooper ME, Parving HH, Shahinfar S, et al. Comparison of different measures of urinary protein excretion for prediction of renal events. J Am Soc Nephrol 2010; 21 (8): 1355-60.

6. Go SA, Chertow GM, Fan D, Mc Culloch CE, Hsu CH-Y. Chronic kidney disease and the risks of death, cardiovascular events and hospitalization. N Engl J Med 2004; 351: 1296-305.

7. Nag S. All-cause and cardiovascular mortality in diabetic subjects increases significantly with reduced estimated glomerular filtration rate (eGFr): 10 years' data from the south Tees Diabetes Mortality study. Diabet Med 2007; 24: $10-7$.

8. Matsushita K, van der Velde M, Astor BC, Woodward M, Levey AS, de Jong PE, et al. Chronic Kidney Disease Prognosis Consortium. Association of estimated glomerular filtration rate and albuminuria with all-cause and cardiovascular mortality in general population cohorts: a collaborative meta-analysis. Lancet 2010 12; 375 (9731): 2073-81.

9. Ficociello LH, Perkins BA, Roshan B, Weinberg JM, Aschengrau A, Warram JH, et al. Renal hyperfiltration and the development of microalbuminuria in type 1 diabetes. Diabetes Care 2009 32: 889-93.

10. Gaede P, Vedel P, Larsen N, Jensen GV, Parving HH, Pedersen O. Multifactorial intervention and cardiovascular disease in patients with type 2diabetes. $\mathrm{N}$ Engl J Med 2003; 348: 383-93.

11. MacIsaac RJ, Tsalamandris C, Panagiotopoulos S, Smith TJ, McNeil KJ, Jerums G. Nonalbuminuric renal insufficiency in type 2 diabetes. Diabetes Care 2004; 27 (1): 195-200.

12. National Kidney Foundation KD. Clinical Practice Guidelines for Chronic Kidney Disease: Evaluation, Classification, and Stratification. Am J Kidney Dis 2002; 39 (suppl 1): S1-S266.

13. Minutolo R, De Nicola L, Mazzaglia G, Postorino M, Cricelli C, Mantovani LG, et al. Detection and awareness 
of moderate to advanced CKD by primary care practitioners: a cross-sectional study from Italy. Am J Kidney Dis. 2008; 52 (3): 444-53.

14. Obrador G, García-García G, Villa A, Rubilar X, Olivera N, Ferreira E, et al. Prevalence of chronic kidney disease in the Kidney Early Evaluation Program (KEEP) México and comparison with KEEP US. Kidney Int 77; S2-S8.

15. Takahashi S, Okada K, Yanai M. The Kidney Early Evaluation Program (KEEP) of Japan: results from the initial screening period. Kidney Int 77; S17-S23.

16. Jones CA, Francis ME, Eberhardt MS, Chavers B, Coresh J, Engelgau M. Microalbuminuria in the US population: third National Health and Nutrition Examination Survey. Am J Kidney Dis 2002; 39 (3): 445-59.

17. Ravera M, Noberasco G, Re M, Filippi A, Gallina AM, Weiss U. Chronic kidney disease and cardiovascular risk in hypertensive type 2 diabetics: a primary care perspective. Nephrol Dial Transplant 2009; 24 (5): 1528-33.

18. Van der Meer V, Wielders HP, Grootendorst DC, de
Kanter JS, Sijpkens YW, Assendelft WJ. Chronic kidney disease in patients with diabetes mellitus type 2 or hypertension in general practice. Br J Gen Pract 2010; 60 (581): 884-90.

19. Jerums G, Premaratne E, Panagiotopoulos S, Clarke S, Power DA, MacIsaac RJ. New and old markers of progression of diabetic nephropathy. Diabetes Res Clin Pract. 2008 13; 82: S30-S7.

20. Tsalamandris C, Allen TJ, Gilbert RE, Sinha A, Panagiotopoulos S, Cooper ME, et al. Progressive decline in renal function in diabetic patients with and without albuminuria. Diabetes 199443 (5): 649-55.

21. Kramer HJ, Nguyen QD, Curhan G, Hsu CY. Renal insufficiency in the absence of albuminuria and retinopathy among adults with type 2 diabetes mellitus. JAMA 2003 25; 289 (24): 3273-7.

22. Carmine Z. Endothelial Dysfunction and the Kidney: Emerging Risk Factors for Renal Insufficiency and Cardiovascular Outcomes in Essential Hypertension. J Am Soc Nephrol 2006; 17: S61-3. 